\section{P88 CELL FREE DNA BACTERIAL SEQUENCES IN PATIENTS WITH LUPUS NEPHRITIS DIFFER FROM PATIENTS WITH IGA NEPHROPATHY AND MEMBRANOUS NEPHROPATHY}

${ }^{1}$ Bartosz Foroncewicz, ${ }^{1,2}$ Krzysztof Mucha, ${ }^{2}$ Arkadiusz Gładki, ${ }^{1}$ Barbara Moszczuk, ${ }^{1}$ Natalia Krata, ${ }^{1}$ Marta Monticolo, ${ }^{2}$ Urszula Zielenkiewicz, ${ }^{2}$ Piotr Zielenkiewicz, 1,2Leszek Pączek. 'Dept. of Immunology, Transplantology and Internal Diseases, Medical University of Warsaw, Warsaw; ${ }^{2}$ Institute of Biochemistry and Biophysics, Polish Academy of Sciences, Warsaw, Poland

10.1136/lupus-2020-eurolupus. 132

Background The circulating free DNA (cfDNA) originating mostly from the abnormal cell apoptosis, necrosis or netosis contains sequences of microorganisms encountered previously by the patients. Therefore, it may be a source of information about past infections and may become a tool to evaluate human microbiome in relation to specific diseases. The aim of the study was to identify bacterial sequences in cfDNA of patients with different types of glomerulopathies.

Methods Blood samples from 9 patients with lupus nephritis (LN), 5 with IgA nephropathy, 4 with membranous nephropathy and 3 healthy controls were collected once. cfDNA was isolated (QIAmp, Qiagen) and quantified (Thermo Fisher Scientific, Waltham). Sequencing libraries were constructed, and quality checked (KAPA-Roche, Basel). Samples were sequence $\mathrm{d}$ on NextSeq 550 (Illumina, San Diego), before a multi-step bioanalysis.

Results Bacterial sequences represented $0.031 \%$ of the cfDNA. The most frequent bacterial genera in the cfDNA of patients with glomerulopathies included: Esherichia, Streptococcus, Klebsiella, Brevundimonas and Moraxella and they varied between the studied patient groups. The cluster of four LN and one MN patients had distinctive bacterial cfDNA pattern which was observed on the species, family, order, class and phylum level.

Conclusions Bacterial sequences in cfDNA of patients with lupus nephritis differ from patients with $\operatorname{IgA}$ nephropathy and membranous nephropathy. Validation in a larger patient population is warranted.

\section{P89 EPIGENOME-WIDE ASSOCIATION STUDY REVEALS DIFFERENTIAL DNA METHYLATION IN SYSTEMIC LUPUS ERYTHEMATOSUS PATIENTS WITH A HISTORY OF ISCHEMIC HEART DISEASE}

\footnotetext{
${ }^{1} J u l i a n a$ Imgenberg-Kreuz, ${ }^{2}$ Christopher Sjöwall, ${ }^{2}$ Martina Frodlund, ${ }^{3}$ Iva Gunnarsson, ${ }^{3}$ Elisabet Svenungsson, ${ }^{1}$ Dag Leonard. 'Dept. of Medical Sciences, Uppsala University, Uppsala; ${ }^{2}$ Dept. of Clinical and Experimental Medicine, Linköping University, Linköping; ${ }^{3}$ Dept. of Medicine Solna, Karolinska Institutet, Karolinska University Hospital, Stockholm, Sweden
}

\subsection{6/lupus-2020-eurolupus. 133}

Background/Purpose Patients with Systemic Lupus Erythematosus (SLE) have an increased risk of ischemic heart disease (IHD). Altered DNA methylation patterns have been reported both in SLE and in individuals with a history of IHD in the general population. We performed a case-case epigenome-wide association study (EWAS) for IHD in patients with SLE to identify phenotype-specific differences in DNA methylation.

Methods DNA methylation profiles from peripheral blood samples of 33 SLE patients with a history of IHD (myocardial infarction and/or angina pectoris) and 66 matched (sex and age) SLE patients without any prior cardiovascular events were generated on the HumanMethylation450k array (Illumina). All patients were female, fulfilled $\geq 4$ ACR-82 SLE criteria and were recruited at the Uppsala, Linköping and Karolinska University hospitals, Sweden. A linear regression model including age at sampling, blood cell type distribution and HM450k BeadChip as covariates was fitted, and association p-values were Bonferroni corrected.

Results We identified 210 differentially methylated CpG sites (DMCs) in SLE IHD, with a majority (84\%) of sites showing decreased methylation levels in IHD. DMCs were annotated to 155 unique genes, of which $64 \%$ were characterized as interferon-induced. DMCs with the largest effect size were located at Complement component 4 binding protein alpha (C4BPA), Membrane spanning 4-domains A3 (MS4A3) and Triggering receptor expressed on myeloid cells 1 (TREM1). Further, a differentially methylated region with multiple DMCs was observed in the promoter region of Programmed cell death 1 gene (PDCD1), where increased methylation was associated with IHD.

Conclusion The results of this study highlight genes and mechanisms that may be implicated in the pathogenesis of and/or recovery from IHD in patients with SLE. Identified DMCs can serve as candidates for functional studies and as potential biomarkers for IHD in patients with SLE.

\section{P90 GENETIC RISK, SMOKING AND THE DEVELOPMENT OF SYSTEMIC AUTOIMMUNE RHEUMATIC DISEASE}

${ }^{1}$ Henrik Christian Bidstrup Leffers, ${ }^{2}$ David Westergaard, ${ }^{2}$ Karina Banasik, ${ }^{1}$ Søren Jacobsen. ${ }^{1}$ Copenhagen Lupus and Vasculitis Clinic, Rigshospitalet, Copenhagen; ${ }^{2}$ Translational Disease Systems Biology, NNF CPR, University of Copenhagen, Copenhagen, Denmark

\subsection{6/lupus-2020-eurolupus. 134}

Purpose Environmental and genetic factors have individually been extensively researched in the pathogenesis of systemic autoimmune rheumatic diseases (SARDs). Notably, more than half of the associations are shared by at least two autoimmune diseases and SLE exhibits association to almost all. Smoking is an overall risk factor for the development of any SARD. We aim to determine a polygenic risk score for SARD including smoking as a covariate which could advocate for gene-environment interaction.

Methods A case-control study will be conducted to determine a polygenic risk score including smoking.

Data will be retrieved from the UK Biobank, which is a general-population cohort of roughly 0.5 million participants recruited across the UK during 2006-2010. Participants have been genotyped. A genome wide association study (GWAS) will be performed using the Scalable and Accurate Implementation of GEeneralized mixed model (SAIGE). Cases are defined to have SARD if having one of the following diagnoses: SLE, rheumatoid arthritis, polymyositis/dermatomyositis, systemic sclerosis or primary Sjögrens syndrome. Controls comprise UK Biobank participants not fulfilling the case definition or having other autoimmune diseases.

Results We have identified 1,048 patients in the UK Biobank with at least one SARD and more than 300,000 matched controls. Results from the initial GWAS calculations are shown in figure 1. Based on a top-100 list of susceptibility SNPs, we will derive a polygenic risk core and determine to which extent smoking, gender and age increase the risk of SARD among subjects which are highly genetically susceptible. 


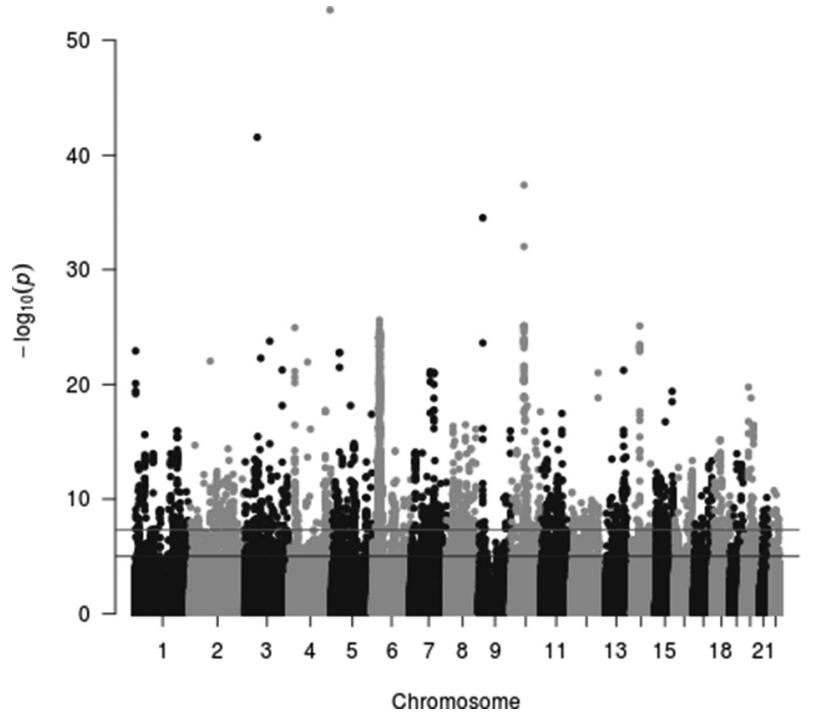

Abstract P90 Figure 1

External validity is tested by replication in independent cohorts from the Danish Blood Donor Study and Copenhagen Biobank.

Conclusion Clinical tools based on genetics predictive of SARDs are in wanting but have generally been judged of little to no useful information. In this study, we will provide a validated predictive model of SARD based on multiple genes and interaction with non-genetic factors.
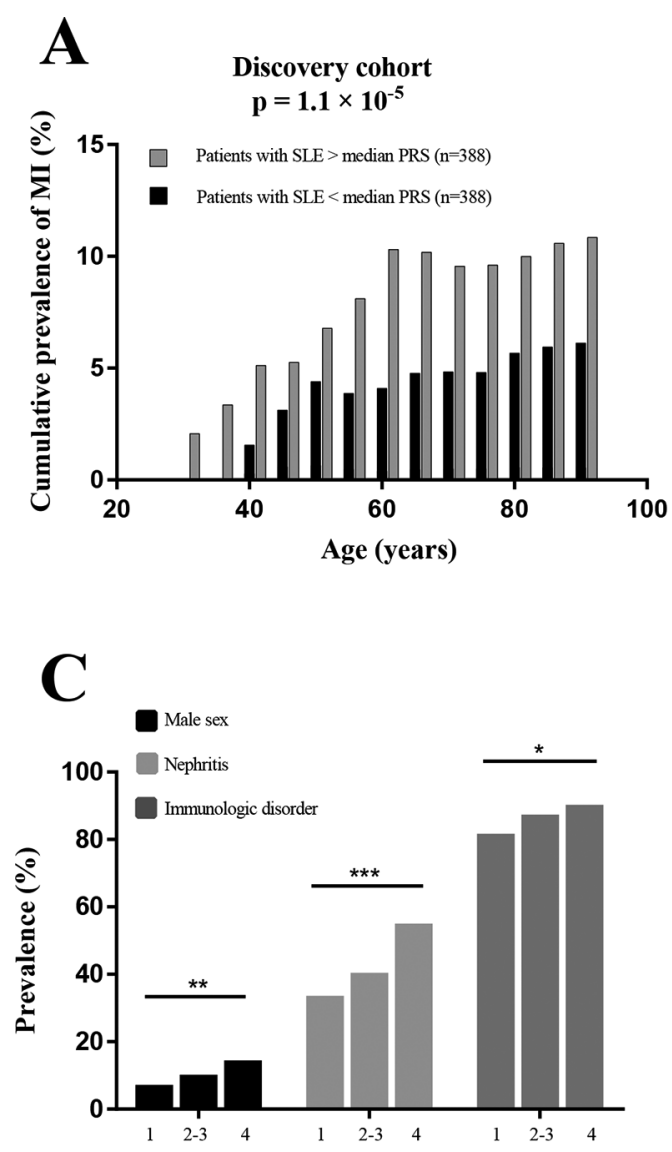

Polygenic risk score quartile
Acknowledgement Supported by the Danish Rheumatism Association

\section{P91 THE DEVELOPMENT AND VALIDATION OF A POLYGENIC RISK SCORE FOR MYOCARDIAL INFARCTION IN SLE}

${ }^{1}$ Sarah Reid, ${ }^{1}$ Johanna K Sandling, ${ }^{1}$ Andrei Alexsson, ${ }^{1}$ Pascal Pucholt, ${ }^{2}$ Christopher Sjöwall, ${ }^{3}$ Karoline Lerang, ${ }^{4}$ Andreas Jönsen, ${ }^{5}$ Iva Gunnarsson, ${ }^{1}$ Ann-Christine Syvänen, ${ }^{6}$ Anne Troldborg, ${ }^{7}$ Anne Voss, ${ }^{4}$ Anders A Bengtsson, ${ }^{3}$ Øywind Molberg, ${ }^{8}$ Søren Jacobsen, ${ }^{5}$ Elisabet Svenungsson, 'Lars Rönnblom, ${ }^{1}$ Dag Leonard. 'Uppsala University, Uppsala; ${ }^{2}$ Linköping University, Linköping, Sweden; ${ }^{3}$ University of Oslo, Oslo, Norway; ${ }^{4}$ Lund University, Lund; ${ }^{5}$ Karolinska Institutet, Solna, Sweden; ${ }^{6}$ Aarhus University, Aarhus; ${ }^{7}$ Odense University Hospital, Odense; ${ }^{8}$ Copenhagen University Hospital, Copenhagen, Denmark

\subsection{6/lupus-2020-eurolupus. 135}

Background Patients with SLE have increased morbidity and mortality due to cardiovascular disease. Here, we construct and validate a polygenic risk score (PRS) for myocardial infarction (MI) in SLE.

Methods Patients with SLE (European decent, $\geq 4$ ACR-criteria) were genotyped using a $200 \mathrm{~K}$ Immunochip SNP array (discovery cohort, Sweden, $\mathrm{n}=776$ ) and custom MassARRAY assays (replication cohort, Norway/Denmark, $n=890$ ). In the discovery cohort, 57 SNPs with previously established association with SLE development $\left(\mathrm{p}<5.0 \times 10^{-8}\right)$ were investigated for associations with MI using a cox regression model. Significant SNPs were included in a PRS, weighted by their ORs for MI development. The PRS was subsequently validated in the replication cohort.
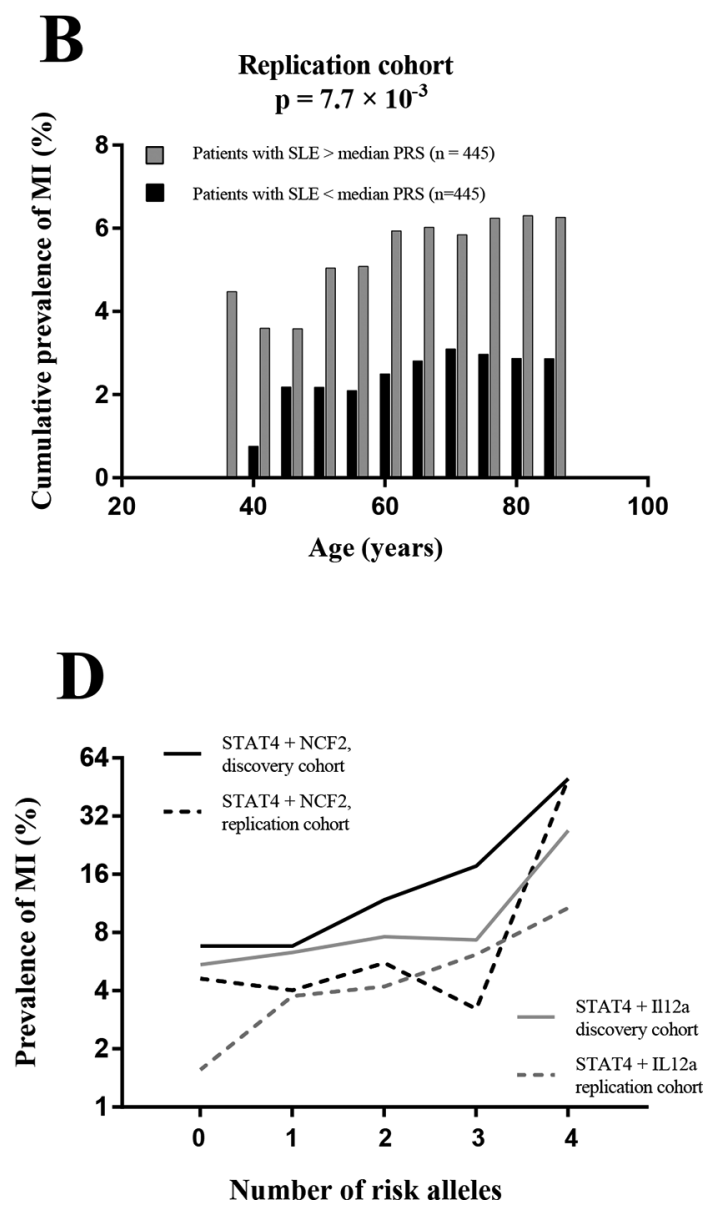